\title{
Mycophenolate therapy in autoimmune pancreatitis
}

\author{
Adlene Jenita Jebakumar, Prabhu Deepak Udayakumar*, Erik J Peterson and Martin L Freeman \\ Divisions of Rheumatology and Gastroenterology, University of Minnesota, USA
}

Autoimmune pancreatitis (AIP) is an uncommon cause of chronic pancreatitis with an estimated prevalence rate of $0.82 / 100,000$ in Japan [1-3]. Exact incidence and prevalence in the United States is unknown. AIP is generally divided into two types: Type 1 (Lymphoplasmacytic sclerosing pancreatitis/IgG4 related) and Type 2 (Idiopathic ductcentric pancreatitis) [4,5]. Systemic glucocorticoids remain the mainstay of treatment for AIP. However, more than $50 \%$ of patients with AIP treated with steroids experience relapse with the steroid taper [6]. Remission maintenance in many patients requires chronic steroid use, which poses risk of adverse effects [7]. In order to minimize risk of chronic steroid use, drugs such as azathioprine (AZA), 6-mercaptopurine (6-MP) and rituximab (RTX) have been used in AIP [7]. Thiopurine Methyl Transferase (TPMT) activity is reduced in about $11 \%$ of general population, who therefore may not tolerate AZA or 6-MP [8]. Also, AZA is associated with drug-induced pancreatitis, which may mimic AIP flare and cause therapeutic confusion. Disadvantages of RTX include its requirement for intravenous infusion and its expense. Hence there is a need for alternative cheaper, effective and safer oral steroid-sparing agents for AIP. Mycophenolate mofetil (MMF), a powerful inhibitor of lymphocyte proliferation, is a commonly used immunosuppressive agent by rheumatologists. It is available in generic form and also the safety profile is known over few decades. It may be better tolerated than AZA or 6-MP in some patients. However data regarding efficacy and safety of MMF in AIP are lacking. As of 4-2016, less than 15 such cases have been reported in Englishlanguage literature [9-12].

AIP patients were identified by Rheumatology and Gastroenterology providers at the University of Minnesota (UMN), a tertiary referral center for patients with pancreatic diseases. On the basis of previous case series [13], UMN providers have used mycophenolate for AIP treatment in recent years. Here we present one center's experience with mycophenolate mofetil in 4 patients with AIP.

Patient A is a 23-year-old female with history of celiac disease, type 1 diabetes mellitus, and chronic urticaria who presented with recurrent abdominal pain. Her abdominal pain was initially thought to be due to sphincter of Oddi dysfunction, but failed to respond to biliary and pancreatic sphincterotomies by endoscopic retrograde cholangio-pancreatography (ERCP). She was started on prednisone $40 \mathrm{mg}$ daily for chronic urticaria of unclear etiology (skin biopsy was non-specific) and the prednisone was quickly tapered off. Surprisingly, her abdominal pain improved dramatically. However, she was not a candidate for long-term prednisone therapy because of underlying type 1 diabetes mellitus and anxiety. MMF (750 mg twice daily) was started for her urticaria. However both urticarial rash and abdominal pain improved. When MMF dose was tapered down, her abdominal pain worsened. EUS showed hyper-echoic foci and lobularity in the pancreas. Ampullary biopsy showed Immunoglobulin G4 (IgG4)positive plasma cells $>30$ high-powered field (hpf) with associated tissue eosinophilia. Serum IgG4 was in normal range. There were no documented side effects. She has continued on MMF for a total of six years with sustained improvement in her abdominal pain.

Patient B is a 20 year-old female who presented with recurrent abdominal pain, nausea and vomiting diagnosed as recurrent acute pancreatitis. CT of the abdomen showed an enlarged sausage-shaped pancreas with hypodense rim and multiple pancreatic duct strictures with a nondilated pancreatic duct strongly suggestive of AIP. Papillary biopsy showed no evidence of IgG4-positive cells. Serum IgG4 level was normal. Prednisone 40mg daily was begun with good response. However she had at least 3 relapses with taper off of prednisone, each of which were treated with prednisone and eventually she became steroid-dependent. Azathioprine 50mg daily was started, but was stopped within 2 weeks due to concern over possible drug-induced pancreatitis. After ERCP with placement of a pancreatic stent through a high-grade stricture, she was started on MMF (1 gram twice daily). Her symptoms rapidly improved, with normalization of pancreatic enzymes. Subsequent ERCP showed complete resolution of pancreatic strictures. Steroids were tapered off successfully over sixteen months. She was continued on MMF for over a year with maintained remission. After discontinuing MMF, she continued without relapse for at least 2 years.

Patient $\mathrm{C}$ is 42 year-old female with a previous history of Sjogren's syndrome featuring sicca symptoms, elevated anti-nuclear antibody, anti-Ro antibody and rheumatoid factor, who presented with recurrent epigastric pain. EUS revealed hyperechoic strands and hyperechoic foci in pancreatic parenchyma. The pancreatic duct had hyperechoic walls and was tortuous/ectatic. Ampullary biopsy showed >10 IgG4 plasma cells per hpf. Serum IgG4 was normal. She was then started on a trial of prednisone (maximum dose $40 \mathrm{mg}$ per day) but the response to steroids over two months was not clear. She was then started on MMF (total of $1250 \mathrm{mg}$ daily). Abdominal pain improved initially and MMF was continued for greater than 4 years. Intermittent minor flares of abdominal pain occurred during MMF therapy.

Patient D is a 41-year-old male with history of Type 2 diabetes mellitus who presented with recurrent abdominal pain associated with significantly elevated ( $>3 \mathrm{X}$ upper limit of normal) lipase and amylase. EUS showed concentric thickening of the pancreatic duct in the genu of the pancreas with loss of duct architecture, body and tail showing changes consistent with chronic pancreatitis. EUS-guided core biopsy showed focal storiform fibrosis with focal mild acute inflammation

Correspondence to: Dr. Prabhu Deepak Udayakumar, Assistant Professor, Division of Rheumatology, University of Minnesota, Tel: 612626 6560; E-mail: pudayaku@umn.edu

Received: June 17, 2016; Accepted: July 25, 2016; Published: July 28, 2016 
within the pancreatic lobular parenchyma. IgG4 immunostain showed only 0-8 IgG4 positive plasma cells per hpf. However, serum IgG4 was markedly elevated at 430 (normal range 11-86). He responded very well to placement of a temporary stent through the pancreatic stricture, followed by 1-month course of tapering prednisone. 2 months after prednisone discontinuation, acute pancreatitis relapsed. AZA $75 \mathrm{mg}$ daily was started; however, smoldering abdominal pain continued. AZA was stopped after several weeks when drug-induced pancreatitis was suspected and MMF $1000 \mathrm{mg}$ twice daily was begun. Prednisone was also restarted at $40 \mathrm{mg}$ daily and tapered off in about 4 months. The patient experienced two flare-ups of pancreatitis (abdominal pain with elevated serum pancreatic enzymes) after the prednisone taper. So MMF dose was increased to $1250 \mathrm{mg}$ twice daily. He was subsequently followed in his home state.

Patient A, C and D presumably had Type 1 AIP, as there was evidence of IgG4 disease, although patient D had features more typically associated with Type 2 AIP, i.e. recurrent acute pancreatitis with ductal strictures. Patient B had features most consistent with Type 2 AIP, although her CT scan was fairly classic for Type 1 AIP with a "sausage" shaped enlarged pancreas with rim enhancement. Increasingly, patients are referred to Rheumatology for collaborative medical management of autoimmune pancreatitis, especially Type 1 AIP associated with IgG4-related disease. Small case series suggest steroid-sparing effects of several agents in AIP [2,6,7]. Though AZA and RTX remain commonly used, our data add to previous case reports that MMF also may be effective as a steroid-sparing agent in AIP [912]. MMF may be an alternative option for patients who cannot receive or are intolerant of RTX or AZA. Also one may prefer MMF to AZA as AZA may cause iatrogenic pancreatitis, which may cause therapeutic confusions. MMF is cheaper than RTX. The safety profile of MMF is known over few decades when compared to RTX, which is used only since about year 2000. However, use of MMF in AIP needs to be formally studied in prospective randomized controlled trials.

\section{Key Message}

Mycophenolate mofetil may be effective in treating patients with autoimmune pancreatitis who cannot receive rituximab or azathioprine.

\section{References}

1. Shimosegawa T, Chari ST, Frulloni L, Kamisawa T, Kawa S, et al. (2011) International consensus diagnostic criteria for autoimmune pancreatitis: guidelines of the International Association of Pancreatology. Pancreas 40: 352-358. [Crossref]

2. Hart PA, Kamisawa T, Brugge WR, Chung JB, Culver EL, et al. (2013) Long-term outcomes of autoimmune pancreatitis: a multicentre, international analysis. Gut 62: 1771-1776. [Crossref]

3. Nishimori I, Tamakoshi A, Otsuki M; Research Committee on Intractable Diseases of the Pancreas, Ministry of Health, Labour, et al. (2007) Prevalence of autoimmune pancreatitis in Japan from a nationwide survey in 2002. J Gastroenterol 42 Suppl 18: 6-8. [Crossref]

4. Klöppel G, Detlefsen S, Chari ST, Longnecker DS, Zamboni G (2010) Autoimmune pancreatitis: the clinicopathological characteristics of the subtype with granulocytic epithelial lesions. J Gastroenterol. 45: 787-793. [Crossref]

5. Zhang L, Chari S, Smyrk TC, Deshpande V, Klöppel G, et al. (2011) Autoimmune pancreatitis (AIP) type 1 and type 2: an international consensus study on histopathologic diagnostic criteria. Pancreas 40: 1172-1179. [Crossref]

6. Sandanayake NS, Church NI, Chapman MH, Johnson GJ, Dhar DK, et al. (2009) Presentation and management of post-treatment relapse in autoimmune pancreatitis/ immunoglobulin G4-associated cholangitis. Clin Gastroenterol Hepatol. 7: 1089-1096. [Crossref]

7. Hart PA, Zen Y, Chari ST (2015) Recent Advances in Autoimmune Pancreatitis. Gastroenterology 149: 39-51. [Crossref]

8. Lennard L, Van loon JA, Weinshilboum RM (1989) Pharmacogenetics of acute azathioprine toxicity: relationship to thiopurine methyltransferase genetic polymorphism. Clin Pharmacol Ther. 46: 149-154. [Crossref]

9. Hart PA, Topazian MD, Witzig TE, Clain JE, Gleeson FC, et al. (2013) Treatment of relapsing autoimmune pancreatitis with immunomodulators and rituximab: the Mayo Clinic experience. Gut 62: 1607-1615. [Crossref]

10. Ghazale A, Chari ST, Zhang L, Smyrk TC, Takahashi N, et al. (2008) Immunoglobulin G4-associated cholangitis: clinical profile and response to therapy. Gastroenterology 134: 706-715. [Crossref]

11. Sodikoff JB, Keilin SA, Cai Q, Bharmal SJ, Lewis MM, et al. (2012) Mycophenolate mofetil for maintenance of remission in steroid-dependent autoimmune pancreatitis. World J Gastroenterol 18: 2287-2290. [Crossref]

12. Mannion M, Cron RQ (2011) Successful treatment of pediatric IgG4 related systemic disease with mycophenolate mofetil: case report and a review of the pediatric autoimmune pancreatitis literature. Pediatr Rheumatol Online J. 9: 1. [Crossref]

13. Fulton B, Markham A (1996) Mycophenolate mofetil. A review of its pharmacodynamic and pharmacokinetic properties and clinical efficacy in renal transplantation. Drugs 51 278-298. [Crossref]

Copyright: (2016 Jebakumar AJ. This is an open-access article distributed under the terms of the Creative Commons Attribution License, which permits unrestricted use, distribution, and reproduction in any medium, provided the original author and source are credited. 